Published in final edited form as:

Curr Opin Neurobiol. 2007 October ; 17(5): 505. doi:10.1016/j.conb.2007.11.006.

\title{
Neuronal and glial cell biology
}

\author{
Ann Marie Craig and \\ Brain Research Centre and Department of Psychiatry, University of British Columbia, 2211 \\ Wesbrook Mall, Koerner F149, Vancouver, BC V6K 1T1, Canada amcraig@interchange.ubc.ca \\ Wieland B Huttner \\ Max Planck Institute of Molecular Cell Biology and Genetics, Pfotenhauerstrasse 108, 01307 \\ Dresden, Germany huttner@mpi-cbg.de
}

\section{Introduction}

Brain development and signaling are intimately linked to the form and function of constituent neuronal and glial cells. Recent advances in microscopy, the major tool of cell biologists, and methods for manipulating molecular signals have made this an area ripe for discovery. This issue of Current Opinion in Neurobiology highlights some recent advances. A focus of this issue is how axonal and dendritic arbors are shaped, directed by intrinsic and extrinsic cues being uncovered by powerful genetic experiments. Remarkably, axonal arbors undergo vigorous structural plasticity even in the adult brain. This is complemented by articles addressing morphogenetic processes involving highly specialized membranes, myelin, and photoreceptive membranes. Studies of fundamental neuronal and glial cell biology can have a major impact on understanding neurological disorders, as exemplified by the articles on opioid receptors in addiction and on a novel neurodegenerative proteinopathy.

\section{Axonal and dendritic arbors: development and adult plasticity}

Numerous families of attractive and repulsive contact-dependent and contact-independent axon guidance molecules have been identified, often initially from cell culture and invertebrate models. Understanding how such cues act together with neuronal activity to guide axon projections in the mammalian brain has best been achieved in the mouse olfactory system, as reviewed by Imai and Sakano. Sensory neurons in the olfactory epithelium express only one of a thousand odorant receptor genes, and sensory neurons expressing the same odorant receptor converge their axons onto the same small subset of glomeruli in the olfactory bulb. The basis for how odorant receptors direct such precise connectivity has been elusive until recently. Partly on the basis of elegant genetic experiments from their own group, Imai and Sakano discuss how axons utilize dorso-ventral positional information, odorant receptormediated cAMP signaling, and classic axon guidance molecules including neuropilins, Kirrels, and ephrin-As and EphAs for precise navigation. A key concept is the idea that odorant receptor function via cAMP and neuronal activity regulate the expression level of specific axon guidance molecules in individual sensory neurons.

Axonal arbors are not fixed in the adult, but rather undergo a remarkable degree of structural plasticity, as revealed in recent studies complementing assays of dendritic spine plasticity. Gogolla, Galimberti, and Caroni discuss the evidence for, mechanisms of, and implications of plasticity of axon sidebranches, en-passant boutons, and terminaux boutons. Although most axon boutons are stable for months, an intermingled proportion depending on the specific cell type and target region form anew or disappear, perhaps undergoing several cycles of remodeling during the lifetime of an organism. 
Remodeling is greatest during crucial periods of development but has also been directly visualized in adult mouse and monkey cortex in vivo by two-photon imaging of GFP-labeled axons. Given the large synaptic sampling territory of an individual axon, such plasticity can significantly alter microcircuitry and is no doubt linked to experience-related learning, memory, and adaptation, as insightfully conveyed by Gogolla, Galimberti, and Caroni.

Less well studied than axon guidance, dendritic morphogenesis equally controls neuronal connectivity. Much of our insight into molecular mechanisms controlling dendrite arborization comes from Drosophila genetic screens and is adeptly reviewed by Gao. As exemplified by mammalian Purkinje neurons and Drosophila dendritic arborization neurons, dendrite arbor is often considered a defining characteristic of a neuron. Thus it is not surprising that transcriptional regulatory networks control many aspects of dendrite arborization. The link between transcriptional networks and surface molecules such as cadherins and semaphorins that regulate dendrite targeting is one active area of investigation. Exciting recent discoveries include the role of Tricornered-kinase/Furry signaling in dendritic tiling, nonredundant coverage by sister neurons, and the role of Down's syndrome cell adhesion molecule (Dscam) homophilic adhesion in selfavoidance, repulsion between sister branches of a single neuron.

\section{Further specialized membranes: myelination and photoreceptor morphogenesis}

Efficient axonal function requires myelination by oligodendroglia in the CNS and Schwann cells in the PNS, exemplifying the intimate contact required between neurons and glia for proper brain function. Simons and Trotter discuss recent progress in our understanding of the biogenesis of the myelin membrane, a specialized subdomain of the oligodendrocyte/Schwann cell plasma membrane. One focus of their review is on the establishment of cell polarity that occurs in the glial cell concomitant with glial-axon recognition. Here, an insightful overview of the molecular players involved is provided. Another focus is on the interaction between the lipids and proteins that are concentrated in myelin, and how these interactions result in the formation of myelin membrane domains during exocytotic and endocytotic membrane trafficking. Simons and Trotter incorporate the recent insight obtained in these areas into an intriguing mechanistic concept about the cell biological basis of myelination.

Photoreceptor cells form another highly specialized membrane which mediates phototransduction. Major insights into the morphogenesis of the photoreceptor membrane have come from Drosophila genetics, as reviewed by Knust. As in the case of myelin-forming glial cells, cell polarity is of key importance for photoreceptor morphogenesis. Thus, a major aspect is the differentiation of the photoreceptor cell apical membrane into the rhabdomeric, lightsensing and the stalk subdomains. The review provides an in-depth discussion of the localization and function of the molecules implicated in photoreceptor, and in particular rhabdomere, morphogenesis. Also here, as a further parallel to myelination, membrane trafficking has emerged as a central mechanism that controls morphogenesis.

\section{Cell biology of neurological diseases: neurodegeneration and addiction}

A second crucial aspect addressed in the review by Knust is the link to retinal degeneration in mammals including humans. Thus, many of the genes that exert crucial roles in photoreceptor morphogenesis in the Drosophila eye have mammalian orthologues, and mutations in the latter result in retinal degeneration.

A different type of neurodegeneration, caused by a novel proteinopathy, is the topic of the review by Forman, Trojanowski, and Lee. Specifically, they concentrate on the nuclear $43 \mathrm{kDa}$ transactive response DNA-binding protein (TDP-43), which was recently identified as the major disease protein in frontotemporal lobar degeneration (FTLD) and amyotrophic lateral sclerosis (ALS). In a comprehensive manner, the review by Forman, Trojanowski, and Lee 
discusses the physiology and pathology of TDP-43, and proposes a classification of neurodegenerative proteinopathies involving TDP-43.

Martini and Whistler discuss another situation where understanding and treating neurological disorders requires understanding fundamental neuronal and glial cell biology. Specifically, Martini and Whistler lead us expertly through the complex issue of how the use of morphine to alleviate chronic pain is complicated by the development of tolerance and dependence. Such systemic adaptations and related behavioral issues are linked at the cellular level to effects on opioid receptor desensitization and endocytosis. Although morphine has good potency and selectivity for opioid receptors, it does not promote endocytosis and recycling, and thus a normal turnover of signaling, as well as endogenous enkephalin ligands. Thus considerations of opioid receptor phosphorylation and arrestin binding are driving the search for more benificient drugs or combinations to alleviate chronic pain.

\section{Conclusions}

Now more than ever, progress in neuroscience requires a multidisciplinary effort. A major goal of cellular neurobiologists is to integrate the molecular and circuit levels, leading to a greater depth of understanding of brain function and behavior. Cellular level changes are at the heart of many neurological disorders and diseases, leading to the development of directed molecular therapies. We look forward to a further acceleration of progress in neuronal and glial cell biology as our abilities to peer into the brain and manipulate cellular signaling continue to improve.

\section{Biography}

Ann Marie Craig is professor and Canada Research Chair in Neurobiology. Her laboratory studies cellular and molecular mechanisms of CNS synapse development and plasticity. Work has focused on sequential assembly and activity-regulated dynamics of molecular components of glutamate synapses, and roles of neurexin and neuroligin cell surface molecules. Current research addresses roles of novel cell surface proteins, molecular assembly of GABAergic synapses, and regulation by local versus global activity, using hippocampal culture and mouse model systems.

Wieland B Huttner is executive director of the Max Planck Institute of Molecular Cell Biology and Genetics and honorary professor of Neurobiology at the University of Technology, Dresden, Germany. He investigates the cell biology of neural stem and progenitor cells in order to understand the evolution of mammalian brains at a molecular level. Current research in his group is focused on neural progenitor cell polarity and cell division, progenitor lineage relationships, and the stem cell membrane microdomain marker prominin-1/CD133. 